\title{
Inaptidão sorológica pelos vírus HCV e HIV em doadores de sangue em Centro de Hemoterapia no nordeste brasileiro
}

\author{
Serological disability due to HCV and HIV viruses in blood donors on brazilian northeast
}

\section{Discapacidad serológica por virus VHC y VIH en donantes de sangre en noreste brasileño}

Paulo Celso Curvelo Santos Junior ${ }^{1 *}$, Weber de Santana Teles ${ }^{1}$, Milenna Freitas Santana ${ }^{1}$, Ruth Cristini Torres ${ }^{1}$, Ângela Maria Melo Sá Barros ${ }^{2}$, Monise Cardoso Alves ${ }^{1}$, Max Cruz da Silva ${ }^{3}$, Isabelle Borges Primo de Carvalho', Rute Nascimento da Silva', Verónica de Lourdes Sierpe Jeraldo.

\begin{abstract}
RESUMO
Objetivo: Explorar alguns aspectos da inaptidão sorológica em doadores de um banco de sangue no nordeste brasileiro entre o período de 2010 a 2017. Métodos: Trata-se um estudo retrospectivo de caráter descritivo e exploratório dos candidatos à doação com sorologia positiva para HIV e HCV, que foi realizada através do programa HEMOPLUS no banco de sangue no nordeste brasileiro Resultados: Foram calculadas prevalências totais e anuais de inaptidão sorológica bem como avaliado o perfil dos candidatos à doação. Dentre 52.201 candidatos à doação no período do estudo, 40.724 (78\%) foram considerados aptos e $1.114(2,7 \%)$ inaptos para doação, sendo que 40 $(3,6 \%)$ foram considerados inaptos por apresentarem sorologia positiva para HCV, e $16(1,4 \%)$ para HIV. Quanto ao perfil dos candidatos à doação, $75,2 \%$ eram do sexo masculino e $48 \%$ tinham entre $30-49$ anos. Conclusão: Houve uma tendência decrescente de inaptidão para HCV e HIV entre os doadores, indicando uma diminuição do risco de contaminação através da transfusão de sangue, destacando a importância da triagem sorológica para garantir a segurança transfusional.
\end{abstract}

Palavras-chave: Transfusão de sangue, HIV, HCV, Prevalência, Testes sorológicos.

\begin{abstract}
Objective: This study aims to explore some aspects of serological disability in donors of a blood bank in northeastern Brazil between 2010 and 2017. Methods: This is a retrospective, descriptive and exploratory study of candidates for donation with positive serology for HIV and HCV, which was carried out through the HEMOPLUS program at the blood bank in northeastern Brazil Results: Total and annual prevalence of serological disability were calculated and the profile of candidates for donation was evaluated. Among 52,201 candidates for donation during the study period, $40,724(78 \%)$ were considered fit and 1,114 (2.7\%) unfit for donation, with $40(3.6 \%)$ being considered unfit for presenting positive serology for HCV, and $16(1.4 \%)$ for HIV. As for the profile of the donation candidates, $75.2 \%$ were male and $48 \%$ were between $30-49$ years old. Conclusion: There was a decreasing trend of inability for HCV and HIV among donors, indicating a decrease in the risk of contamination through blood transfusion, highlighting the importance of serological screening to ensure transfusion safety.
\end{abstract}

Keywords: Blood transfusion, HIV, HCV, Prevalence, Serological problems.

\section{RESUMEN}

Objetivo: Este estudio tiene como objetivo explorar algunos aspectos de la discapacidad serológica en donantes de un banco de sangre en el noreste de Brasil entre 2010 y 2017. Métodos: Se trata de un estudio retrospectivo, descriptivo y exploratorio de candidatos a donación con serología positiva para VIH y VHC, que se llevó a cabo a través del programa HEMOPLUS en el banco de sangre del noreste de Brasil. Resultados: Se calculó la prevalencia total y anual de discapacidad serológica y se evaluó el perfil de los candidatos a donación. Entre 52.201 candidatos a donación durante el período de estudio, 40.724 (78\%) se consideraron aptos y $1.114(2,7 \%)$ no aptos para la donación, siendo $40(3,6 \%)$ no aptos para apresentar serología positiva para VHC y $16(1,4 \%)$ para el VIH. En cuanto al perfil de los candidatos a donación, el 75,2\% eran hombres y el $48 \%$ tenían entre 30 y 49 años. Conclusión: Hubo una tendencia decreciente de incapacidad para el VHC y el VIH entre los donantes, lo que indica una disminución en el riesgo de contaminación por transfusión de sangre, lo que destaca la importancia del cribado serológico para garantizar la seguridad de la transfusión.

Palabras clave: Transfusión de sangre, VIH, VHC, Prevalencia, Pruebas serológicas.

${ }^{1}$ Universidade Tiradentes (Unit), Aracajú - SE. *E-mail: paulo.curvelo.jr@gmail.com

${ }^{2}$ Universidade Federal do Rio de Janeiro (UFRJ), Rio de Janeiro - RJ.

${ }^{3}$ Faculdade Piodécimo de Canindé de São Francisco (FAPIDE), Canindé de São Francisco - SE.

SUBMETIDO EM: 4/2021

ACEITO EM: 5/2021

PUBLICADO EM: 5/2021 


\section{INTRODUÇÃO}

O vírus da hepatite $C$ pertence ao gênero Ácido Hepacivirus, família Flaviviridae, que se distribui amplamente pelo mundo se tornando responsável pela cronicidade da doença. O HCV é transmitido principalmente por sangue contaminado, através de agulhas e seringas no compartilhamento para uso de drogas injetáveis, acidentes com material biológico, transfusão de concentrado de hemácias, hemoderivados e por contato sexual com pessoas infectadas pelo vírus (JOSAHKIAN JA, et al., 2011).

No cenário atual o Egito é o país mais acometido pelo vírus da hepatite $C$, tendo por volta de $15 \%$ dos habitantes contaminados, sendo que a fundamental causa é o uso de agulhas no recurso terapêutico da esquistossomose. Em relação ao continente africano, o maior indícice verifica-se no território central e occidental. Na américa Latina e Caribe a estimative é que 7 a 9 milhões de indivíduos estejam infectados pelo virus da hepatite C. Na África, a maior prevalência ocorre nas Regiões Central, Ocidental e Subsaariana. Nos países da América Latina e Caribe, estima-se que existam entre 7 e 9 milhões de adultos positivos para antiHCV, no tempo em que nações da América do Norte, apresentam baixos indíces do virus da hepatite $\mathrm{C}$ (OLIVEIRA TJB, et al., 2018).

A taxa de infecção do virus da hepatite B no território brasileiro a aproximadamente de 1,4 a 2,5\%17. Durante os anos de 1999 a 2011 foram notificados 82.041 , destes $75 \%$ se encontra entre 30 a 59 anos, sendo o maior índice os indíviduos do sexo masculine (OLIVEIRA TJB, et al., 2018).

O quadro clínico decorrente da infecção pelo HCV na maioria das vezes cursa de forma assintomática, sendo que manifestações clinicas podem aparecem de 6 a 7 semanas após a exposição ao vírus, evoluindo para a sua forma crônica em $80 \%$ a $90 \%$ dos casos. Na forma crônica, o quadro clínico pode evoluir para cirrose e hepatocarcinoma, principalmente em pacientes imunossuprimidos. Os exames realizados na constatação da proteína própria do anti-HCV demonstram que esse tipo de vírus é o mais encontrado após transfusão (COSTA AG, et al., 2012).

Por outro lado, o Vírus da Imunodeficiência Humana (HIV), causador da AIDS, é um retrovírus pertencente à família Retrovidae. Em razão das modificações genéticas existem dois tipos de HIV, 1 e 2, o HIV tipo 1 é encontrado atualmente em todos os continentes, sendo o mais patogênico, e o HIV tipo 2 que tem ocorrência mais restrita ao continente africano, nesse contexto os dois possuem a mesma ação no organismo do ser humano, entretanto o HIV-1 produz menos partículas virais que o HIV-1. No Brasil há o predomínio do HIV-1, sendo que o registro de infecções pelo HIV tipo 2, é pouco frequente (SCHUSTER AD, 2013).

O HIV é uma doença que pode ser transmitida por meio do contato sexual sem o uso de preservativo, além do contato direto com sangue contaminado, incluindo o compartilhamento de agulhas e de seringas, transfusão de sangue/hemoderivados, bem como acidentes com material biológico. Além disso, a transmissão ocorre de forma vertical, no qual a mãe trasmite para o bebê durante a gestação, no momento do parto ou na amamentação (RIBEIRO ATB e JACOCIUNAS LVA, 2016).

O processo de triagem clínica em banco de sangue não é considerado suficiente para garantir uma transfusão segura, mesmo com a existência do voto de auto exclusão. Durante a entrevista clínica pode haver omissão de informações consideradas importantes como uso de medicamentos e doenças crônicas em curso ou ainda desconhecimento em relação ao fato de ser portador de alguma doença. Desta forma, se torna obrigatória à realização de testes laboratoriais sorológicos de alta sensibilidade e especificidade em todas as doações de sangue, com o objetivo de garantir uma transfusão segura (LIMA TC e SOTTA DE, 2015).

A Portaria no 158, de 04 de fevereiro de 2016, que redefine o regulamento técnico de procedimentos hemoterápicos e a Resolução de Diretoria Colegiada (RDC) - $n^{\circ} 34$ da Agência Nacional de Vigilância Sanitária (ANVISA) de 2014, dispõem sobre as Boas Práticas no ciclo do sangue e determinam os exames sorológicos a serem realizados para o diagnóstico de infecções como: sífilis, doença de Chagas, hepatite $B$, hepatite C, HIV 1 e 2 e Vírus T-linfotrópico Humano (HTLV) I e II, malária, e o citomegalovírus humano (HCMV). A realização destes testes é fundamental para que a bolsa de sangue coletada seja liberada para transfusão. Além da sorologia de anticorpos HIV e triagem clínica, a aplicação do Teste de Ácido Nucleico (NAT) foi implantada visando aumentar a segurança de quem está recebendo o sangue, pois consegue detectar Ácido Desoxirribonucleico (DNA) e RNA de agentes infecciosos presentes no sangue no início do processo infeccioso, ou seja, quando ainda não há resposta imunológica (WENDEL S, et al., 2014). 
O ato transfusional é utilizado como mecanismo de tratamento para indivíduos que sofrem de anemias, talassemias, hemofilias, leucemias e também como terapia em processos cirúrgicos e em transplantes, portanto, os hemocomponentes e seus derivados devem atender o sistema de gestão da qualidade para 0 uso humano (SILVA NV, et al., 2013).

Para que aconteça a transmissão de agentes patogênicos pela transfusão sanguínea é necessário que o candidato à doação de sangue seja portador do agente infeccioso em seu sangue, e que as análises realizadas na triagem não sejam capazes de detectá-lo Carrazzone CFV, et al. (2010). Este fato pode ocorrer no período denominado de janela imunológica, que corresponde ao período compreendido entre o momento que se dá a introdução do agente infeccioso no hospedeiro suscetível e o início da detecção dos anticorpos produzidos frente aos antígenos ou a detecção desses antígenos. Dessa forma, durante a fase de janela imunológica, um doador poderá ter em seu sangue um número considerável de partículas virais na ausência de marcadores sorológicos ou sinais e sintomas de uma infecção em curso, porém, ainda não apresentar níveis detectáveis, se encaixando nos resultados de exames falsos negativos. O que poderá ocasionar a contaminação do receptor (PETRY A, 2013).

Procedimentos para a transfusão de sangue requerem medidas de atenção em relação aos testes falsonegativos, sendo assim se faz necessário a utilização de testes mais sensíveis e específicos para a detecção de anticorpos ou antígenos no período de janela imunológica (SILVA NV, et al., 2013).

O Enzyme Linked Immunosorbent Assay (ELISA), e a quimioluminescência são os testes mais utilizados nos serviços de hemoterapia, pois permitem boa reprodutibilidade, fácil execução e automação, tendo como objetivo a detecção de doadores não aptos por conta da presença de algum agente infeccioso em seu sangue (WENDEL S, et al., 2014).

A sensibilidade do teste ELISA é dada pela detecção de anticorpos anti-HIV em 22 dias após a infecção e anti-HCV em aproximadamente 60 dias. Sendo estes testes classificados como de triagem, pois tem como função detectar todos os indivíduos infectados, produzindo poucos resultados falso-negativos (JOSAHKIAN JA, et al., 2004).

A constante evolução dos conjuntos diagnósticos através de novas pesquisas e descobertas acarretou no desenvolvimento/aprimoramento de testes sorológicos para detecção de anticorpos formados pelo sistema imunológico após a infecção, seguidos de testes para identificação de antígenos e, no final da década de 90, o surgimento dos testes moleculares (NAT) capazes de reduzir o período de janela imunológica. Para o HCV a diminuição é altamente significativa (72\%) passando a ser de aproximadamente 15 dias; para o HIV, o decréscimo é de $50 \%$, fazendo com que a janela imunológica seja de aproximadamente 10 dias (PEREIRA AMB e NASCIMENTO PRF, 2004).

Tendo em vista a importância da triagem nos bancos de sangue o presente trabalho teve como objetivo a avaliação da inaptidão de doação com sorologia positiva para Hepatite C e HIV em um centro de Hemoterapia no nordeste brasileiro no período compreendido entre janeiro de 2010 e janeiro de 2017.

\section{MÉTODOS}

Foi realizado um estudo seccional retrospectivo dos candidatos à doação com sorologia positiva para HIV e HCV no banco de sangue no nordeste brasileiro. Foram incluídos os doadores na faixa etária de 18 a 65 anos que compareceram ao Instituto de Hematologia e Hemoterapia de Sergipe no período de janeiro de 2010 a janeiro 2017, conforme legislação vigente.

Todas as informações foram coletadas a partir do banco de dados disponibilizado pelo sistema gerenciador do Laboratório de Análises Clínicas (LIAC) central sorológica com parceira do Ministério da Saúde, localizado na cidade do Rio de Janeiro/RJ.

A coleta de dados foi realizada utilizando um programa específico para bancos de sangue denominado Hemoplus (Sistema de Gestão hemoterápica), no qual constam os relatórios estatísticos de doações por faixa etária, gênero, sorologias positivas e causas de rejeições na triagem clínica. 
Trabalho aprovado por meio do parecer consubstanciado 2.954.787. Os dados foram analisados a partir da ferramenta de estatística Excell, em relação à prevalência de doações com sorologia positiva, negativa e indeterminada para HIV e HCV e determinado o perfil dos candidatos inaptos em relação ao gênero, faixa etária e sorologia para as infecções virais em estudo. Os resultados foram apresentados em gráficos e tabelas.

\section{RESULTADOS}

No período estudado de janeiro 2010 a janeiro 2017, no banco de sangue do nordeste brasileiro, foram registrados 52.201 candidatos à doação de sangue, dos quais 40.724 (78\%) foram liberados para doação e $11.477(22 \%)$ foram descartados na triagem clínica.

Das 40.724 doações liberadas, $1.114(2,7 \%)$ foram consideradas inaptas por apresentarem sorologia positiva para alguma doença infectocontagiosa. Destes últimos, $16(1,4 \%)$ foram considerados inaptos por apresentarem sorologia positiva para HIV e $40(3,6 \%)$ positivas para o vírus HCV (Tabela 1).

Tabela 1 - Índice de inaptidão clínica e sorológica (total e para HCV e HIV) de 52.201 candidatos à doação no banco de sangue do nordeste brasileiro, de janeiro 2010 a janeiro 2017.

\begin{tabular}{ccc}
\hline Parâmetro & № & $\%$ \\
\hline Candidatos à doação & 52.201 & 100 \\
Inaptos clínicos & 11.477 & 22 \\
Aptos clínicos & 40.724 & 78 \\
Inaptos sorológicos & 1.114 & 2,7 \\
Inaptos HCV & 40 & 3,6 \\
Inaptos HIV & 16 & 1,4 \\
\hline
\end{tabular}

Fonte: Junior PCCS, et al.,2021.

Na Tabela 2 são apresentados os dados de distribuição das doações aptas e inaptas em relação aos parâmetros sócios demográficos de gênero e faixa etária. Foi observado um predomínio de doações inaptas do gênero masculino e na faixa etária $\geq 30$ anos.

Tabela 2 - Distribuição dos doadores inaptos por sorologia positiva ou indeterminada para HCV e HIV, de acordo com o perfil sócio demográfico (Gênero/Faixa etária), no período de 2010 a 2017.

\begin{tabular}{lcccccc}
\hline \multirow{2}{*}{ Categoria } & Doadores aptos & $\begin{array}{c}\text { Doações Inaptas HCV } \\
\mathbf{2}^{\circ} \text { amostra }\end{array}$ & $\begin{array}{c}\text { Doações Inaptas HIV } \\
\mathbf{2}^{\circ} \text { amostra }\end{array}$ \\
\cline { 2 - 7 } Gênero & № & $\%$ & № & $\%$ & № & $\%$ \\
\cline { 2 - 7 } & & & & & & 1.34 \\
\hline Masculino & 30.641 & 75.2 & 33 & 2.96 & 1 & 0.08 \\
\hline Feminino & 10.083 & 24.6 & 7 & 0.62 & 10 & 0.89 \\
\hline 30-49 anos & 19.561 & 48 & 18 & 1.61 & 10 & \\
\hline
\end{tabular}

Fonte: Junior PCCS, et al.,2021.

A Tabela 3 mostra o quantitativo de doações com sorologia positiva para HIV junto ao resultado realizado para uma segunda amostra no período de 2010-2017. Observa-se uma baixa constante no número de 
doações ao longo do período, destas doações, 37 foram reagentes para HIV, no entanto somente 16 doadores voltaram para realização do teste para a segunda amostra. No período 2014-2015 foi verificado o maior número de amostras positivas para HIV apesar do número menor de doadores de anos anteriores.

Tabela 3 - Ocorrência anual de doações reagentes (sorologia positiva ou indeterminada) para o vírus HIV, no período de 2010 a 2017.

\begin{tabular}{cccccc}
\hline Período & $\begin{array}{c}\text { Doações } \\
\text { testadas }\end{array}$ & $\begin{array}{c}\mathbf{1}^{\circ} \text { amostra } \\
\mathbf{( N )}\end{array}$ & $\begin{array}{c}\mathbf{1}^{\circ} \text { amostra } \\
(\%)\end{array}$ & $\begin{array}{c}\mathbf{2}^{\circ} \text { amostra } \\
(\mathbf{N})\end{array}$ & $\begin{array}{c}\mathbf{2}^{\circ} \text { amostra } \\
(\%)\end{array}$ \\
\hline $2010-2011$ & 5.238 & 9 & 0,17 & 3 & 0,05 \\
$2011-2012$ & 7.248 & 3 & 0,04 & 2 & 0,02 \\
$2012-2013$ & 7.166 & 4 & 0,05 & 2 & 0,02 \\
$2013-2014$ & 6.130 & 8 & 0,13 & 3 & 0,04 \\
$2014-2015$ & 5.838 & 10 & 0,17 & 4 & 0,06 \\
$2015-2016$ & 4.607 & 1 & 0,02 & 0 & 0 \\
$2016-2017$ & 4.497 & 2 & 0,04 & 2 & 0,04 \\
\hline Total & 40.724 & 37 & 0,62 & 16 & 0,23 \\
\hline
\end{tabular}

Fonte: Junior PCCS, et al., 2021.

Em relação a sorologia para HCV, observa-se uma diminuição progressiva de amostras inaptas para doação, com exceção do período 2014-2015. Da mesma forma que para HIV, existe uma quantidade expressiva de doadores que não voltam para realizar a coleta de segunda amostra para novos testes (Tabela 4).

Tabela 4 - Ocorrência anual de doações reagentes por sorologia positiva ou indeterminada para o vírus HCV, no período de 2010 a 2017.

\begin{tabular}{cccccc}
\hline \multirow{2}{*}{ Período } & $\begin{array}{c}\text { Doações } \\
\text { testadas }\end{array}$ & $\begin{array}{c}\mathbf{1}^{\circ} \text { amostra } \\
\mathbf{( N )}\end{array}$ & $\begin{array}{c}\mathbf{1}^{\circ} \text { amostra } \\
(\%)\end{array}$ & $\begin{array}{c}\mathbf{2}^{\circ} \text { amostra } \\
(\mathbf{N})\end{array}$ & $\mathbf{2}^{\circ}$ amostra (\%) \\
\hline $2010-2011$ & 5.238 & 38 & 0,72 & 20 & 0,38 \\
$2011-2012$ & 7.248 & 10 & 0,13 & 5 & 0,06 \\
$2012-2013$ & 7.166 & 5 & 0,06 & 1 & 0,01 \\
$2013-2014$ & 6130 & 6 & 0,09 & 3 & 0,04 \\
$2014-2015$ & 5838 & 12 & 0,20 & 10 & 0,17 \\
$2015-2016$ & 4607 & 6 & 0.13 & 1 & 0,02 \\
$2016-2017$ & 4497 & 0 & 0 & 0 & 0 \\
\hline Total & $\mathbf{4 0 . 7 2 4}$ & $\mathbf{7 7}$ & $\mathbf{1 , 3 3}$ & $\mathbf{4 0}$ & $\mathbf{0 , 6 8}$ \\
\hline
\end{tabular}

Fonte: Junior PCCS, et al.,2021. 
Observa-se a maior prevalência de doadores inaptos para HCV no ano de 2010-2011 com 20 (0,38\%) das doações, os doadores inaptos para HIV foram de 4 (0,6\%) no ano de 2014-2015 (Figura 1). Tal fato pode ser analisado pela diminuição de doadores que voltaram para realizar os testes da sua segunda amostra, já que o número total de amostras analisadas nesse período foram de 40 para HCV em 2010-1011 e de 16 para HIV em 2014-1015.

Figura 1 - Distribuição do número de candidatos a doação de sangue inaptos na segunda amostra por sorologia positiva para HIV e HCV, no período de 2010-2017

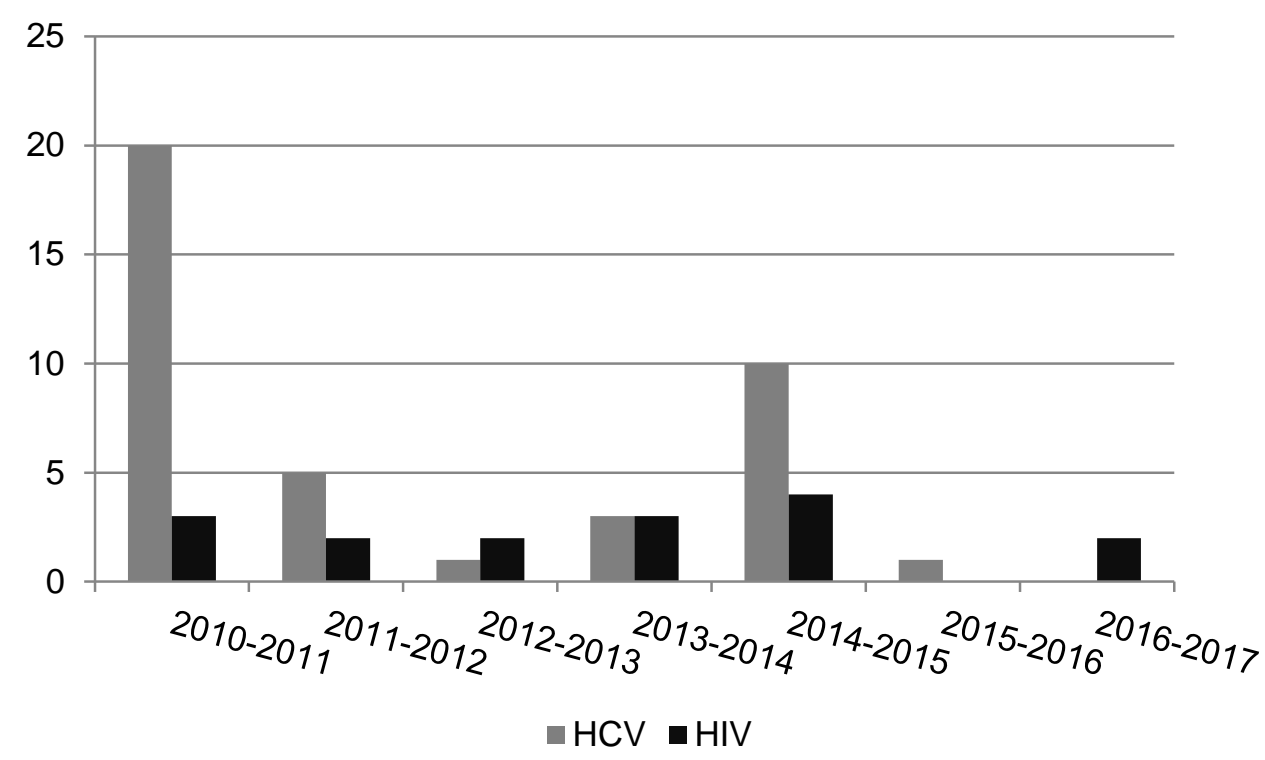

Fonte: Junior PCCS, et al.,2021.

\section{DISCUSSÃO}

De acordo com a Portaria $n^{\circ}$ 158, de 4 de fevereiro de 2016, que redefine o regulamento técnico de procedimentos hemoterápicos, afirma que na triagem clínica a inaptidão do candidato a doação de sangue pode ser transitória ou permanente. Entre as fundamentais causas para inaptidão permanentes estão o etilismo crônico, neoplasias malignas, doenças de coração graves, o diabetes mellitus, a doença de Chagas, a tuberculose, a hepatite viral após 10 anos de idade, as infecções pelo vírus da hepatite $\mathrm{B}$, da hepatite $\mathrm{C}$, HIV, HTLV I/II, a epilepsia e a sífilis recorrente. Em relação as causas de inaptidão transitórias apresentamse a anemia, o aborto, a acupuntura, o uso de piercing e/ou de tatuagem, as alergias, a amamentação, a cefaleia, as cirurgias em geral e a gravidez (BRASIL, 2016).

O predomínio das infecções que são transmitidas pelo sangue, impossibilitando o candidato a doar sangue tem decrescido imensamente nos últimos anos, devido a evolução da medicina transfusional, entretanto pode haver perigos de transmissão em que a medicina transfusional tem evoluído e se aprimorado, mas o risco de infecção continua existindo. Assim sendo, investigações que realizem avaliação da incidência das doenças infecciosas nos indivíduos que doam sangue, torna-se de suma importância para prevenir a transmissão e ponderar serventia dos programas de controle destas patologias (SULTAN S, et al., 2018).

A taxa de descarte sorológico não caracteriza a prevalência de uma determinada infecção na população de doadores de sangue, entretanto retrata um conjunto de variáveis que tem extrema importância para garantir a qualidade do sangue, e que é assegurado através da triagem sorológica para evitar a transmissão de doenças infecciosas. $O$ índice de inaptos por sorologia positiva encontrado no presente estudo foi menor que o observado por Martins APB, et al. (2015), onde o estudo apresentou (4,5\%) de sorologia positiva, no estudo em questão foi evidenciado $(2,7 \%)$, sendo (3,59\%) para HVC e $(1,43 \%)$ para HIV. 
O estudo mostrou que a maioria dos indivíduos que doaram sangue no centro de Hemoterapia do nordeste brasileiro era do sexo masculino Santos EA, et al. (2018) em um estudo realizado em outro Instituto foram encontrados valores similares, mostrando que $84,08 \%$ dos doadores eram do sexo masculino e $15,92 \%$ do sexo feminino. Este grande contingente de doadores do sexo masculino provavelmente está influenciando também nos maiores índices de inaptidão sorológica para HIV e HVC analisadas no estudo, ressaltando que o número de indivíduos com sorologia positiva para HIV foi o dobro quando comparado como sexo feminino.

Estes resultados sorológicos que mostram maior número de homens com sorologia positiva para HIV 15 $(1,34 \%)$ e HCV $33(2,96 \%)$ podem estar relacionados a aspectos comportamentais tais como o uso de drogas injetáveis, promiscuidade, não uso de preservativos em relações sexuais, fatores que predispõem a um risco maior de adquirir estas infecções. Estudo realizado por Martins APB, et al. (2015) em outro Instituto localizado na Regional de Cruz Alta-Rio Grande do Sul, mostraram que esses fatores estão totalmente relacionados ao alto índice de homens com sorologia positiva para doenças infecciosas.

Nos últimos anos houve um aumento do número de infecção pelo HIV em indivíduos do sexo feminino, em regiões em desenvolvimento, é caracterizada por uma propensão em que no início acometia a população masculina e passou a crescer na população feminina, gerando um crescimento da transmissão vertical. (FRIEDRICH L, et al., 2016).

Com relação à faixa etária, verificou-se maior número de doadores com sorologia reagente tanto para HCV $(1,61 \%)$ quanto para HIV $(0,89 \%)$ com faixa etária entre 30 e 49 anos. Os dados mostram-se semelhantes àqueles descritos em estudo realizado por Josahkian JA, et al. (2010) que relataram uma frequência de $9,1 \%$ dos doadores com idade igual ou superior a 30 anos com sorologia reagente para HIV e HCV, no Hemocentro Regional de Uberaba-MG, Fundação Hemominas.

Os resultados obtidos pelo estudo mostram uma discrepância entre os valores reagentes em primeira e segunda amostra, isso pode ser explicado pelo não comparecimento dos doadores ao banco de sangue para realização da coleta de segunda amostra para realização de novos testes. Outro fator que está relacionado à divergência de números reagentes em segunda amostra é o teste falso positivo, sugere-se estar relacionado ao uso de medicamentos, infecções em curso e metodologias empregadas na avaliação do sangue (FRIEDRICH L, et al., 2016).

Com relação ao HCV entre os anos de 2010-2011 e 2014-2015, foram observados os maiores índices quantitativos de doações com sorologia positiva para HCV testadas em segunda amostra; resultados similares foram encontrados no estudo de Valente VB, et al. (2005) em doadores do Hemocentro de Ribeirão Preto. Nesse mesmo intervalo de estudo, foram verificadas doações reagentes em primeira amostra $(0,65)$, porém não confirmadas devido ao não comparecimento dos doadores para realização de testes em segunda amostra, esta discrepância entre os valores de doações reagentes em primeira e segunda amostra pode também estar ligada a exames falsos positivos devido à omissão de informações, como por exemplo, o uso de medicações e infecções em curso.

Entre os anos de 2013-2014 e 2014-2015 foram observados os maiores índices de doadores com sorologia positiva para HIV testados tanto na primeira como segunda amostra, entretanto $(0,39 \%)$ dos doadores convocados não compareceram para realização dos testes em segunda amostra sendo assim bloqueados no sistema para doações enquanto não realizarem os testes específicos para descartar qualquer hipótese diagnostica dos devidos vírus reagentes nos testes realizados de triagem sorológica.

Após realização de testes em segunda amostra, e os testes mostrando-se reagentes, os doadores são convocados ao local da doação para encaminhamento aos centros de tratamento de doenças infecciosas, onde irão realizar testes específicos para confirmar a sorologia positiva do devido vírus e iniciar o tratamento da devida patologia. Mensalmente, são enviados relatórios de sorologia para os órgãos de notificação compulsória para atualização do sistema quanto ao índice de sorologia positiva nos diferentes municípios.

No setor de banco de sangue, a o sistema de gestão de qualidade e a segurança na transfusão do sangue são fatores importantes e demanda muitos recursos financeiros, informações semelhantes são apresentadas em estudo de descarte sorológico, apontam por grandes valores financeiros utilizados na triagem sorológica 
e do descarte das bolsas de sangue positivas para infecções Aguiar DS, et al. (2018). O descarte das bolsas com sorologia positivas, além dos gastos financeiros que sofrem os centros de hemoterapia que geram esses serviços, a diminuição dos hemocomponentes para as empresas, impacta no atendimento aos pacientes ne carece de transfusões. Por isso faz-se necessário entender o descarte da bolsa positiva no ciclo da doação de sangue, tem em vista que o quantitativo de insumos que são dispensados, auxiliaria na terapia de vários indivíduos visto que cada bolsa pode ser fracionada em vários tipos de hemocomponentes (SALAZAR JM, et al., 2017).

Diante do exposto, a triagem sorológica realizada nos centros de hemoterapia é considerada sensível quanto a confirmação e positividade para doenças testadas, servindo para eliminar bolsas potencialmente infectantes, evitando a contaminação de um possível receptor desse sangue. Desta forma, os doadores que apresentam sorologia positiva para algum dos vírus testados convocados através de uma carta que é de responsabilidade do setor de hemovigilância, assim são orientados a fazer um teste com uma segunda amostra para que seja confirmada a possibilidade de uma infecção junto à triagem clínica, através de técnicas diagnósticas de alta especificidade.

\section{CONCLUSÃO}

Diante desta pesquisa, foi observado que os doadores do sexo masculino apresentaram maior taxa de inaptidão sorológica, podendo ser justificado pelo comportamento de risco. Outros fatores relevantes são as relações de transfusão de sangue, as patologias com alto índice epidemiológico necessitam de atenção especial com serviços de saúde devido a sua evolução silenciosa, evidenciando um diagnóstico precoce afim de evitar a transmissão e aumentar a melhor eficácia ao tratamento. Conclui-se o conhecimento acerca do perfil sorológico dos candidatos a doação de sangue auxilia como ferramenta de melhoria no sistema de gestão da qualidade do hemocentro, atendimento da na triagem clínica e na laboratorial, assim como campanhas de sensibilização a população acerca das doenças transmissíveis, com isso otimizando os recursos disponibilizados pelo Sistema Único de Saúde a ser aplicados no banco de sangue.

\section{REFERÊNCIAS}

1. AGUIAR DS, et al. Enfermagem frente à testagem rápida de sífilis, hiv e hepatites virais em uma comunidade periférica de Macapá, Amapá. Brazilian Journal of health Review, 2018; 1(1): 164-84.

2. BARBAN GB. Importância do teste do ácido nucleico(nat) nos bancos de sangue do brasil. Academia de Ciência e Tecnologia são Jose do Rio Preto-SP. Brazilian Journal of health Review, 2010; 8(3): 1-9.

3. BRASIL. Legislação em Vigilância Sanitária. Resolução RDC $n^{\circ} 34$, de 11 de junho de 2014. Disponível em: https://inc.saude.gov.br/download/resolucao-rdc-n-34-de-11-de-junho-de-2014.pdf. Acessado em: 24 de Abril de 2021.

4. CARRAZZONE CFV, et al. Importância da avaliação sorológica pré-transfusional em receptores de sangue. Revista Brasileira de Hematologia e Hemoterapia, 2004; 26(2): 93-98.

5. COSTA AG et al. Incidência de hepatite c em doadores de sangue do município de Anápolis no ano de 2010 . Revista Ciências da Saúde, 2013, 11(1): 11-17.

6. FERREIRA O. Estudo de doadores de sangue com sorologia reagente para hepatites B e C, HIV sífilis no Hemocentro de Ribeirão Preto. Revista Ciências da Saúde, 2007; 1(9): 90-106.

7. FRIEDRICH L, et al. Transmissão vertical do HIV: uma revisão sobre o tema Vertical transmission of HIV: a review. Revista Científica Pediátrica, 2016; 05(3): 81-6.

8. GONÇALVES KI, et al. Soroprevalência de HIV-1/2 entre doadores de sangue de Goiânia-Goiás. Revista Brasileira de Cardiologia, 2006, 38(4); 263-266.

9. JOSAHKIAN JÁ, et al. Prevalência de inaptidão sorológica pelo Vírus HCV em doadores de sangue no Hemocentro regional de Uberaba-MG, Fundação Hemominas. Revista de Patologia Tropical, 2010; 39(4): 261-272.

10. LIMA TC e SOTTA DE. Avaliação de inaptidão sorológica e molecular pós-implantação da técnica de amplificação de ácidos nucléicos(nat) na triagem da hepatite c e do hiv no centro de hematologia e hemoterapia do paraná - hemepar. Revista Brasileira de Cardiologia, 2015, 8(3): 1-9.

11. MARTINS APB, et al. Soroprevalência de doenças infecciosas em doadores de sangue do Hemocentro Regional de Cruz Alta-Rio Grande do Sul. Revista Clínica de Biomedicina, 2015; 35(4): 211-216.

12. NASCIMENTO AB, et al. Análises parasitológicas de hortaliças comercializadas na feira livre do Ver-o-Peso, Belém PA. Revista Eletrônica Acervo Saúde, 2020; 41(6): 488-499. 
13. OLIVEIRA TJB, et al. Perfil epidemiológico dos casos de hepatite $C$ em um hospital de referência em doenças infectocontagiosas no estado de Goiás, Brasil. Revista Panamericana de Saúde, 2018; 9(1): 51-57.

14. PEREIRA AMB e NASCIMENTO FRF. Prevalência de HIV entre doadores de sangue no banco de sangue do Maranhão. Revista Brasileira Doenças Sexualmente Transmissíveis, 2004; 16(4): 11-13.

15. PETRY A. Implantação dos testes de amplificação de ácidos nucléicos HIV/HCV Bio-Manguinhos na triagem de doadores de sangue: questões epidemiológicas e logísticas. Tese de Doutorado (Doutorado em Ciências da Saúde) Universidade Federal de Santa Catarina, Florianópolis, 2013; 156p.

16. RIBEIRO ATB e JACOCIUNAS LVA. coinfecção sífilis/HIV e sua importância no rastreamento sorológico em bancos de sangue. Biomed Research International, 2016; 36(2): 101-109.

17. RODRIGUEZ AMX, et al. Prevalence of major diseases investigated in the serological screening in units of a blood center. Revista Ciência e Saberes - Facema, 2018; 4(1): 835-840.

18. RODRIGUEZ DM, et al. Prevalência de marcadores sorológicos no Banco De Sangue de Caxias do Sul-RS. Revista Panamericana de Infectologia, 2008; 10(3): 32-35.

19. SALAZAR JM, et al. Seroprevalence, cost per donation and reduction in blood supply due to positive and indeterminate results for infectious markers in a blood bank in Lima, Peru. Revista Brasileira de HematolHogia e Hemoterapia, 2017; 39(2): 102-107.

20. SANTOS EA, et al. Avaliação Epidemiológica das rejeições dos doadores de sangue no HEMOLACEN/SE no período de 2004 a 2006. Revista Brasileira de Cardiologia, 2008; 40(4): 251-256.

21. SCHUSTER AD. Avaliação sorológica de HIV por técnicas de Elisa de quarta geração. Revista Epidemiológica de Controle Infectológico, 2013; 3(4): 112-113.

22. SILVA NV, et al. Métodos alternativos à transfusão de sangue: vantagens e desvantagens. Revista Eletrônica Interdisciplinar, 2013, 1(9): 90-106.

23. SULTAN S, et al. AsmaRehan, Syed Mohammed Irfan SM. Internal quality control of blood products: An experience from a tertiary care hospital blood bank from Southern Pakistan. Biomed Research International, 2018; $10(1): 64$.

24. VALENTE VB, et al. Marcadores sorológicos das hepatites B e C em doadores de sangue do Hemocentro de Ribeirão Preto, SP. Revista da Sociedade Brasileira de Medicina Tropical. 2005; 38(6): 488-492.

25. WENDEL LS, et al. Introdução do NAT no Brazil: algumas considerações adicionais. Revista Brasileira de Hematologia e Hemoterapia, 2009; 31(2): 112-113. 